\title{
Banach-Saks Type and Gurariǐ Modulus of Convexity of Some Banach Sequence Spaces
}

\author{
Henryk Hudzik, ${ }^{1}$ Vatan Karakaya, ${ }^{2}$ Mohammad Mursaleen, ${ }^{3}$ and Necip Simsek ${ }^{4}$ \\ ${ }^{1}$ Faculty of Mathematics and Computer Science, Adam Mickiewicz University, Umultowska 87, 61-614 Poznań, Poland \\ ${ }^{2}$ Department of Mathematical Engineering, Yildiz Technical University, Davutpasa Campus, Esenler, 34750 Istanbul, Turkey \\ ${ }^{3}$ Department of Mathematics, Aligarh Muslim University, Aligarh 202002, India \\ ${ }^{4}$ Department of Mathematics, Istanbul Ticaret University, Üsküdar, Istanbul, Turkey
}

Correspondence should be addressed to Henryk Hudzik; hudzik@amu.edu.pl

Received 5 August 2013; Accepted 4 October 2013; Published 26 March 2014

Academic Editor: Marek Wisla

Copyright (C) 2014 Henryk Hudzik et al. This is an open access article distributed under the Creative Commons Attribution License, which permits unrestricted use, distribution, and reproduction in any medium, provided the original work is properly cited.

Banach-Saks type is calculated for two types of Banach sequence spaces and Gurariǐ modulus of convexity is estimated from above for the spaces of one type among them.

\section{Introduction}

Recently, there has been a lot of interest in investigating geometric properties of sequence spaces besides topological and some other usual properties. In the literature, there are many papers concerning geometric properties of various Banach sequence spaces. For example, geometry of Orlicz spaces and of Musielak-Orlicz spaces has been studied in [125]. Several authors including Cui and Hudzik [26-29], Cui and Meng [30], Suantai [31], and Lee [32] investigated the geometric properties of Cesàro sequence space ces $(p)$. Also Cesàro-Orlicz sequence spaces equipped with Luxemburg norm have been studied in [5,33-36]. Additionally, geometry of Orlicz-Lorentz sequence spaces and of generalized OrliczLorentz sequence space were studied in $[37,38]$. Furthermore, Mursaleen et al. [39] studied some geometric properties of normed Euler sequence space. Additionally, Hudzik and Narloch [40] have studied relationships between monotonicity and complex rotundity properties with some consequences. Besides, some geometrical properties of CalderonLozanovskii sequence spaces have been investigated in [4143].

Quite recently, Karakaya [20] defined a new sequence space involving lacunary sequence space equipped with the Luxemburg norm and studied Kadec-Klee $(H)$ and rotundity
$(R)$ properties of these spaces. Further information on topological and geometric properties of sequence spaces can be found in $[39,44-62]$.

Let $X$ be a real Banach space and $S(X)$ and $B(X)$ be the unit sphere and the unit ball of $X$, respectively. Let $\ell^{0}, c_{0}, c$, $\ell_{\infty}$, and $\ell_{1}$ be the spaces of all real sequences, null, convergent, and bounded sequences and absolutely convergent series, respectively, and let $c_{00}$ be the space of those real sequences which have only a finite number of nonzero coordinates and $\ell_{p}=\left\{x=x(i): \sum_{i=1}^{\infty}|x(i)|^{p}<\infty\right\}$.

Note that $c_{0}, c$, and $\ell_{\infty}$ are Banach spaces with the supnorm $\|x\|_{\infty}=\sup _{i}|x(i)|$ and $\ell_{p}(1 \leq p<\infty)$ are Banach spaces with the norm $\|x\|_{p}=\left(\sum|x(i)|^{p}\right)^{1 / p}$, while $c_{00}$ is not a Banach space with respect to any norm.

Let us recall that a sequence $\{v(i)\}_{i=1}^{\infty}$ in a Banach space $X$ is called Schauder basis of $X$ (or basis for short) if for each $x \in X$ there exists a unique sequence $\{\lambda(i)\}_{i=1}^{\infty}$ of scalars such that $x=\sum_{i=1}^{\infty} \lambda(i) v(i)$, that is, $\lim _{n \rightarrow \infty} \sum_{i=1}^{n} \lambda(i) v(i)=x$.

A sequence space $X$ with a linear topology is called a $K$ space if each of the projection maps $P_{j}: X \rightarrow \mathbb{C}$ defined by $P_{j}(x)=x(i)$ for $x=(x(i))_{i=1}^{\infty} \in X$ is continuous for each natural $j$. A Fréchet space is a complete metric linear space and the metric is generated by an $F$-norm and a Fréchet space which is a $K$-space is called an $F K$-space; that is, a $K$-space $X$ 
is called an $F K$-space if $X$ is a complete linear metric space. In other words, $X$ is an $F K$-space if $X$ is a Fréchet space with continuous coordinate projections. All the sequence spaces mentioned above are $F K$ spaces except the space $c_{00}$.

An $F K$-space $X$ which contains the space $c_{00}$ is said to have the property $A K$ if, for every sequence $\{x(i)\} \in X, x=$ $\sum_{i=1}^{\infty} x(i) e(i)$, where $e(i)=(0,0, \ldots, 1(i$ th-place $), 0,0, \ldots)$. The spaces $c_{0}$ equipped with the sup-norm, $\ell_{p}(1 \leq p<$ $\infty)$ equipped with the norm $\|x\|=\left(\sum_{i=1}^{\infty}|x(i)|^{p}\right)^{1 / p}$ and $\ell^{0}$ endowed with the metric $d(x, y)=\sum_{i=1}^{\infty} 2^{-i}(|x(i)-y(i)| /(1+$ $|x(i)-y(i)|))$ have property $A K$, while $c$ and $\ell_{\infty}$ do not have property $A K$. Banach spaces with continuous coordinate projections are called $B K$-spaces.

A Banach space $X$ is said to be a Köthe sequence space (see $[28,63])$ if $X$ is a subspace of $\ell^{0}$ such that

(i) if $x \in \ell^{0}, y \in X$, and $|x(i)| \leq|y(i)|$, for all $i \in \mathbb{N}$, then $x \in X$ and $\|x\| \leq\|y\|$

(ii) there exists an element $x \in X$ such that $x(i)>0$ for all $i \in \mathbb{N}$.

We say that $x \in X$ is order continuous if, for any sequence $\left(x_{n}\right)$ in $X_{+}$(the positive cone in $\left.X\right)$ such that $x_{n}(i) \leq|x(i)|$ and $x_{n}(i) \rightarrow 0(n \rightarrow \infty)$ for each $i \in \mathbb{N}(n \rightarrow \infty)$, we have $\left\|x_{n}\right\| \rightarrow 0(n \rightarrow \infty)$ holds.

A Köthe sequence space $X$ is said to be order continuous that if all sequences in $X$ are order continuous. It is easy to see that $x \in X$ is order continuous if and only if $\|(0,0, \ldots, 0, x(n+$ $1), x(n+2), \ldots) \| \rightarrow 0$ as $n \rightarrow \infty$.

A Köthe sequence space $X$ is said to have the Fatou property if, for any real sequence $x \in \ell^{0}$ and any $\left\{x_{n}\right\}$ in $X$ such that $x_{n} \uparrow x$ coordinatewisely and $\sup _{n}\left\|x_{n}\right\|<\infty$, we have the fact that $x \in X$ and $\left\|x_{n}\right\| \rightarrow\|x\|$.

A Banach space $X$ is said to have the BanachSaks property if every bounded sequence $\left\{x_{n}\right\}$ in $X$ admits a subsequence $\left\{z_{n}\right\}$ such that the sequence $\left\{t_{k}(z)\right\}$ is convergent in $X$ with respect to the norm, where

$$
t_{k}(z)=\frac{1}{k}\left(z_{1}+z_{2}+\cdots+z_{k}\right), \quad \forall k \in \mathbb{N} .
$$

A Banach space $X$ is said to have the weak Banach-Saks property whenever given any weakly null sequence $\left\{x_{n}\right\}$ in $X$ there exists its subsequence $\left\{z_{n}\right\}$ such that the sequence $\left\{t_{k}(z)\right\}$ converges to zero strongly.

Given any $p \in(1, \infty)$, we say that a Banach space $(X$, $\|\cdot\|)$ has the Banach-Saks property of type $p$ if there exists a constant $c>0$ such that every weakly null sequence $\left\{x_{k}\right\}$ has a subsequence $\left\{x_{k_{\ell}}\right\}$ such that (see [22])

$$
\left\|\sum_{\ell=1}^{n} x_{k_{\ell}}\right\| \leq c n^{1 / p} \quad(\forall n \in \mathbb{N}) .
$$

The Banach-Saks property of type $p \in(1, \infty)$ and the weak Banach-Saks property for Cesàro sequence spaces have been considered in [28]. These properties and stronger property $\left(S_{p}\right)$ for Musielak-Orlicz and Nakano sequence spaces have been studied in [17].

We say that a Banach space $X$ has the weak fixed point property if every nonexpansive self-mapping defined on a nonempty weakly compact convex subset $A$ of $X$ has a fixed point in $A$.

In [64], Garcia-Falset introduced the following coefficient for a Banach space $(X,\|\cdot\|)$ :

$$
\begin{aligned}
R(X)=\sup \left\{\liminf _{n \rightarrow \infty}\left\|x_{n}-x\right\|: x \in B(X)\right. \\
\left.\left\{x_{n}\right\} \subset B(X), x_{n} \longrightarrow 0 \text { weakly }\right\}
\end{aligned}
$$

and he proved (see $[64,65]$ ) that a Banach space $X$ with $R(X)<2$ has the weak fixed point property.

Clarkson modulus of convexity of a normed space $(X,\|\cdot\|)$ is defined (see Clarkson [66] and Day [67]) by the formula

$$
\begin{aligned}
& \delta_{X}(\varepsilon) \\
& \quad=\inf \left\{1-\frac{\|x+y\|}{2} ; x, y \in S(X),\|x-y\|=\varepsilon\right\}
\end{aligned}
$$

for any $\varepsilon \in[0,2]$. The inequality $\delta_{X}(\varepsilon)>0$ for all $\varepsilon \in(0,2]$ characterizes the uniform convexity of $X$ and the equality $\delta_{X}(2)=1$ characterizes strict convexity (=rotundity) of $X$.

The Gurarii modulus of convexity of a normed space $X$ is defined (see $[68,69])$ by

$$
\begin{array}{r}
\beta_{X}(\varepsilon)=\inf \left\{1-\inf _{\alpha \in[0,1]}\|\alpha x+(1-\alpha) y\| ;\right. \\
x, y \in S(X),\|x-y\|=\varepsilon\}
\end{array}
$$

for any $\varepsilon \in[0,2]$. It is obvious that $\delta_{X}(\varepsilon) \leq \beta_{X}(\varepsilon)$ for any Banach space $X$ and any $\varepsilon \in[0,2]$. It is also known that $\beta_{X}(\varepsilon) \leq 2 \delta_{X}(\varepsilon)$ for any $\varepsilon \in[0,2]$ and that $X$ is rotund if and only if $\beta_{X}(\varepsilon)=2$ and as well as that $X$ is uniformly convex if and only if $\beta_{X}(\varepsilon)>0$ for any $\varepsilon \in[0,2]$. Gurariǐ [68] proved that if $X=c_{0}$ is renormed by the norm

$$
\|x\|=\|x\|_{\infty}+\left(\sum_{n=0}\left(\frac{P x_{n}}{2^{n}}\right)^{2}\right)^{1 / 2}, \quad \forall\left\{x_{n}\right\} \in c_{0},
$$

then $\beta_{X}(\varepsilon)=0$ for any $\varepsilon \in[0,2)$ and $\beta_{X}(2)=1$.

Gurarii and Sozonov [70] proved that a normed linear space $(X,\|\cdot\|)$ is an inner product space if and only if, for every $x, y \in S(X)$

$$
\inf _{\alpha \in[0,1]}\|\alpha x+(1-\alpha) y\|=\frac{\|x+y\|}{2} .
$$

Zanco and Zucchi [71] showed an example of a normed space $X$ with $\delta_{X}(2) \neq \beta_{X}(2)$.

Now, we will define Köthe sequence spaces $m(\phi, p)$ and $\ell_{p}(u, v)$ that will be considered in this paper.

Let $\mathscr{C}$ denote the set whose elements are finite sets of distinct positive integers. Given any element $\sigma$ of $\mathscr{C}$, we denote by $c(\sigma)$ the sequence $\left\{c_{n}(\sigma)\right\}$ such that $c_{n}(\sigma)=1$ for $n \in \sigma$, and $c_{n}(\sigma)=0$ otherwise. Further, we define

$$
\mathscr{C}_{\tau}=\left\{\sigma \in \mathscr{C}: \sum_{n=1}^{\infty} c_{n}(\sigma) \leq \tau\right\}
$$


that is, $\mathscr{C}_{\tau}$ is the set of those $\sigma$ whose support has cardinality at most $\tau$. Let us define

$$
\begin{gathered}
\Phi=\left\{\phi=\left\{\phi_{n}\right\}_{n=1}^{\infty} \in \ell^{0}: \phi_{1}>0,\right. \\
\left.\Delta \phi_{k} \geq 0, \Delta\left(\frac{\phi_{k}}{k}\right) \leq 0, \forall k \in \mathbb{N}\right\},
\end{gathered}
$$

where $\Delta \phi_{n}=\phi_{n}-\phi_{n-1}$.

Given any $\phi \in \Phi$, we define the following sequence space, introduced in [55]:

$$
\begin{aligned}
& m(\phi) \\
& \quad=\left\{x=\left\{x_{n}\right\}_{n=1}^{\infty} \in \ell^{0}: \sup _{\tau \geq 1}\left(\sup _{\sigma \in \mathscr{C}_{\tau}}\left(\frac{1}{\phi_{\tau}} \sum_{n \in \sigma}\left|x_{n}\right|\right)\right)<\infty\right\} .
\end{aligned}
$$

Sargent [55] established the relationship of this space to the space $\ell_{p}(1 \leq p \leq \infty)$ and characterized some matrix transformations. In [49], matrix classes $(X, m(\phi))$ have been characterized, where $X$ is assumed to be any $F K$-space.

Recently in [52], some of the geometric properties of $m(\phi)$ have been investigated. In [61], Tripathy and Sen extended the space $m(\phi)$ to $m(\phi, p)$ as follows:

$$
\begin{aligned}
& m(\phi, p) \\
& :=\left\{x=\left\{x_{n}\right\}_{n=1}^{\infty} \in \ell^{0}: \sup _{\tau \geq 1}\left(\sup _{\sigma \in \mathscr{C}_{\tau}}\left(\frac{1}{\phi_{\tau}} \sum_{n \in \sigma}\left|x_{n}\right|^{p}\right)\right)<\infty\right\},
\end{aligned}
$$

for $\phi \in \Phi$ and $p>0$.

It has been proved in [61] that, for $1 \leq p<\infty, m(\phi, p)$ is a Banach space if it is endowed with the norm

$$
\|x\|_{m(\phi, p)}=\sup _{\tau \geq 1}\left(\sup _{\sigma \in \mathscr{C}_{\tau}}\left(\frac{1}{\phi_{\tau}} \sum_{n \in \sigma}\left|x_{n}\right|^{p}\right)^{1 / p}\right),
$$

and that one has the following.

(i) If $\phi_{n}=1$, for all $n \in \mathbb{N}$, then $m(\phi, p)=\ell_{p}$. Moreover, $\ell_{p} \subseteq m(\phi, p) \subseteq \ell_{\infty}$.

(ii) If $p=1$, then $m(\phi, p)=m(\phi)$. Also, for any $p \geq$ $1, m(\phi) \subseteq m(\phi, p)$.

(iii) $m(\phi, p) \subseteq m(\psi, p)$ if and only if $\sup _{\tau \geq 1}\left(\phi_{\tau} / \psi_{\tau}\right)<\infty$.

It is easy to see that $m(\phi, p)$ is a Köthe sequence space, indeed a $B K$-space with respect to its natural norm (see [55]). Note that throughout the present paper we will study the space $m(\phi, p)$ except the case $\phi_{n}=n$, for which it is reduced to the space $\ell^{\infty}$.

Now we will introduce the space $\ell_{p}(u, v)$. Let $u=\left\{u_{n}\right\}_{n=0}^{\infty}$ and let $v=\left\{v_{n}\right\}_{n=0}^{\infty}$ be arbitrary real sequences with all coordinates $u_{k}$ and $v_{k}$ different from zero and let, for any $p \in[1, \infty)$,

$$
\begin{aligned}
& \ell_{p}(u, v) \\
& \quad=\left\{x=\left\{x_{k}\right\}_{k=1}^{\infty}: \sum_{n=1}^{\infty}\left|\sum_{k=1}^{n} u_{n} v_{k} x(k)\right|^{p}<\infty\right\} .
\end{aligned}
$$

It is obvious that this is a linear space. It is known (see [48]) that the functional

$$
\|x\|_{\ell_{p}(u, v)}=\left(\sum_{n}\left|\sum_{k=1}^{n} u_{n} v_{k} x(k)\right|^{p}\right)^{1 / p}
$$

is a norm in $\ell_{p}(u, v)$ and that the couple $\left(\ell_{p}(u, v),\|\cdot\|_{\ell_{p}(u, v)}\right)$ is a Banach space. The space $\ell_{p}(u, v)$ is a generalization of three spaces. Namely, one has the following.

(i) If $\left(v_{k}\right)=(1,1,1, \ldots)$ and $\left(u_{n}\right)=(1 / n)$, then $\ell_{p}(u, v)$ is the Cesáro sequence space $X_{p}$ of nonabsolute type (see [32]) and $\|x\|_{\ell_{p}(u, v)}=\|x\|_{X_{p}}$.

(ii) Let $\left\{p_{n}\right\}$ be a real sequence with $p_{1}>0$ and $p_{n} \neq 0$ for all $n \in \mathbb{N}$. If $\left(v_{k}\right)=\left\{p_{k}\right\}$ and $\left(u_{n}\right)=\left(1 / Q_{n}\right)$, where $Q_{n}=p_{1}+p_{2}+\cdots+p_{n}$ for any $n \in \mathbb{N}$. Then we obtain that $\ell_{p}(u, v)$ is the Riesz sequence space of nonabsolute type denoted by $\gamma^{p}(v)$ and that $\|x\|_{\ell_{p}(u, v)}=\|x\|_{\gamma^{p}(v)}($ see $[44])$.

(iii) Let $\left\{p_{n}\right\}$ be a real sequence with $p_{1}>0$ and $p_{n} \neq 0$, for all $n \in \mathbb{N}, n \geq 2$. If $\left(v_{k}\right)=\left(p_{n-k+1}\right)$ and $\left(u_{n}\right)=$ $\left(1 / Q_{n}\right)$, where $Q_{n}=p_{n}+p_{n-1}+\cdots+p_{1}$ for any $n \in \mathbb{N}$. Then $\ell_{p}(u, v)$ reduces to the Nörlund sequence space of nonabsolute type denoted by $N_{p}$ and $\|x\|_{\ell_{p}(u, v)}=$ $\|x\|_{N_{p}}($ see $[60])$.

\section{Banach-Saks Type of Sequence Space $m(\phi, p)$}

In this section, we investigate some properties of the space $m(\phi, p)$ such as the Fatou property, the Banach-Saks property of type $p$, and the weak fixed point property. Let us start with the following lemma.

Lemma 1. If a BK-space $X$ containing $c_{00}$ has the property $A K$, then it is order continuous, that is, $\|(0,0, \ldots, x(n), x(n+$ $1), \ldots) \| \rightarrow 0$ as $n \rightarrow \infty$ for any $x \in X$.

Proof. From the definition of property $A K$, we have that every $x=\{x(i)\} \in X$ has the unique representation $x=\sum_{i=1}^{\infty} x(i) e(i)$, that is, $x^{[n]}=\sum_{i=1}^{n} x(i) e(i) \rightarrow x$ as $n \rightarrow \infty$. Hence $\left\|x-x^{[n]}\right\|_{X} \rightarrow 0$ as $n \rightarrow \infty$, that is, $\|(0,0, \ldots, x(n), x(n+1), \ldots)\| \rightarrow 0$ as $n \rightarrow \infty$, which means that $X$ is order continuous.

Corollary 2. The space $m(\phi, p)$ is order continuous.

Proof. It is easy to see that $m(\phi, p)$ contains $c_{00}$ and that every $x=\{x(i)\} \in m(\phi, p)$ has the unique representation $x=$ $\sum_{i=1}^{\infty} x(i) e(i)$, that is, $x^{[n]}=\sum_{i=1}^{n} x(i) e(i) \rightarrow x$ as $n \rightarrow \infty$, which means that $m(\phi, p)$ has the property $A K$. Hence, from the above lemma, $m(\phi, p)$ is order continuous.

Theorem 3. The space $m(\phi, p)$ has the Fatou property.

Proof. Let $\mathrm{x}$ be any real sequence from $\left(l^{0}\right)_{+}$and $\left\{x_{n}\right\}$ be any non-decreasing sequence of non-negative elements from $m(\Phi, p)$ such that $x_{n}(i) \rightarrow x(i)$ as $n \rightarrow \infty$ coordinatewisely and $\sup _{n}\left\|x_{n}\right\|_{m(\Phi, p)}<\infty$. 
Let us denote $s=\sup _{n}\left\|x_{n}\right\|_{m(\Phi, p)}$. Then, since the supremum is homogeneous, we have

$$
\begin{aligned}
& \frac{1}{s_{\tau}} \sup _{\tau \geq 1}\left(\sup _{\sigma \in \mathscr{C}_{\tau}}\left(\frac{1}{\Phi_{\tau}} \sum_{i \in \sigma}\left|x_{n}(i)\right|^{p}\right)^{1 / p}\right) \\
& \quad=\sup _{\tau \geq 1}\left(\sup _{\sigma \in \mathscr{C}_{\tau}}\left(\frac{1}{\Phi_{\tau}} \sum_{i \in \sigma}\left|\frac{x_{n}(i)}{s}\right|^{p}\right)^{1 / p}\right) \\
& \leq \sup _{\tau \geq 1}\left(\sup _{\sigma \in \mathscr{C}_{\tau}}\left(\frac{1}{\Phi_{\tau}} \sum_{i \in \sigma}\left|\frac{x_{n}(i)}{\left\|x_{n}\right\|_{m(\Phi, p)}}\right|^{p}\right)^{1 / p}\right) \\
& \quad=\frac{1}{\left\|x_{n}\right\|_{m(\Phi, p)}}\left\|x_{n}\right\|_{m(\Phi, p)}=1 .
\end{aligned}
$$

Moreover, by the assumptions that $\left\{x_{n}\right\}$ is non-decreasing and convergent to $x$ coordinatewisely and by the Beppo-Levi theorem, we have

$$
\begin{aligned}
\frac{1}{s^{n}} & \lim _{n \rightarrow \infty}\left[\sup _{\tau \geq 1}\left(\sup _{\sigma \in \mathscr{C}_{\tau}}\left(\frac{1}{\Phi_{\tau}} \sum_{i \in \sigma}\left|x_{n}(i)\right|^{p}\right)^{1 / p}\right)\right] \\
= & \sup _{\tau \geq 1}\left(\sup _{\sigma \in \mathscr{C}_{\tau}}\left(\frac{1}{\Phi_{\tau}} \sum_{i \in \sigma}\left|\frac{x(i)}{s}\right|^{p}\right)^{1 / p}\right) \\
= & \left\|\frac{x}{s}\right\|_{m(\Phi, p)} \leq 1
\end{aligned}
$$

whence

$$
\|x\|_{m(\Phi, p)} \leq s=\sup _{n}\left\|x_{n}\right\|_{m(\Phi, p)}=\lim _{n \rightarrow \infty}\left\|x_{n}\right\|_{m(\Phi, p)}<\infty .
$$

Therefore, $x \in m(\Phi, p)$. On the other hand, since $0 \leq$ $x_{n} \leq x$ for any natural number $n$ and the sequence $\left\{x_{n}\right\}$ is non-decreasing, we obtain that the sequence $\left\{\left\|x_{n}\right\|_{m(\Phi, p)}\right\}$ is bounded from above by $\|x\|_{m(\Phi, p)}$. In consequence $\lim _{n \rightarrow \infty}\left\|x_{n}\right\|_{m(\Phi, p)} \leq\|x\|_{m(\Phi, p)}$, which together with the opposite inequality proved already, yields that $\|x\|_{m(\Phi, p)}=$ $\lim _{n}\left\|x_{n}\right\|_{m(\Phi, p)}$.

Theorem 4. The space $m(\phi, p)$ has the Banach-Saks property of the type $p$.

Proof. Let $\left\{\epsilon_{n}\right\}$ be a sequence of positive numbers with $\sum_{n=1}^{\infty} \epsilon_{n} \leq 1 / 2$. Let $\left\{x_{n}\right\}$ be a weakly null sequence in $B(m(\phi, p))$. Let us set $x_{0}=0$ and $z_{1}=x_{n_{1}}=x_{1}$. Then, there exists $\tau_{1} \in \mathbb{N}$ such that

$$
\left\|\sum_{i \in s_{1}} z_{1}(i) e(i)\right\|_{m(\phi, p)}<\epsilon_{1},
$$

where $s_{1}$ consist of the elements of $\sigma$ which exceed $\tau_{1}$. Since $x_{n} \stackrel{\omega}{\rightarrow} 0$ implies that $x_{n} \rightarrow 0$ coordinatewise, there is $n_{2} \in \mathbb{N}$ such that

$$
\left\|\sum_{i=1}^{\tau_{1}} x_{n}(i) e(i)\right\|_{m(\phi, p)}<\epsilon_{1}
$$

for all $n \geq n_{2}$. Set $z_{2}=x_{n_{2}}$. Then there exists $\tau_{2}>\tau_{1}$ such that

$$
\left\|\sum_{i \in s_{2}} z_{2}(i) e(i)\right\|_{m(\phi, p)}<\epsilon_{2},
$$

where $s_{2}$ consist of all elements of $\sigma$ which exceed $\tau_{2}$. Using again the fact that $x_{n} \rightarrow 0$ coordinatewise, there exists $n_{3}>$ $n_{2}$ such that

$$
\left\|\sum_{i=1}^{\tau_{2}} x_{n}(i) e(i)\right\|_{m(\phi, p)}<\epsilon_{2}
$$

for all $n \geq n_{3}$.

Continuing this process, we can find two increasing sequences $\left\{\tau_{j}\right\}$ and $\left\{n_{j}\right\}$ such that

$$
\begin{gathered}
\left\|\sum_{i=1}^{\tau_{j}} x_{n}(i) e(i)\right\|_{m(\phi, p)}<\epsilon_{j}, \quad \text { for each } n \geq n_{j+1}, \\
\left\|\sum_{i \in s_{j}} z_{j}(i) e(i)\right\|_{m(\phi, p)}<\epsilon_{j},
\end{gathered}
$$

where $z_{j}=x_{n_{j}}$ and $s_{j}$ consist of the elements of $\sigma$ which exceed $\tau_{j}$. Since $\epsilon_{j-1}+\epsilon_{j}<1$, we have

$$
\left(\frac{1}{\phi_{\tau}} \sum_{n \in \sigma}\left|z_{j}(n)\right|\right) \leq \epsilon_{j-1}+\epsilon_{j}<1,
$$

for all $j \in \mathbb{N}$ and $\tau \geq 1$. Hence

$$
\begin{aligned}
\| \sum_{j=1}^{n} z_{j} & \| \sum_{m(\phi, p)}\left(\sum_{i=1}^{\tau_{j-1}} z_{j}(i) e(i)+\sum_{i=\tau_{j-1}+1}^{\tau_{j}} z_{j}(i) e(i)\right. \\
= & \left.+\sum_{i \in s_{j}} z_{j}(i) e(i)\right) \|_{m(\phi, p)} \\
\leq & \left\|\sum_{j=1}^{n}\left(\sum_{i=1}^{\tau_{j-1}} z_{j}(i) e(i)\right)\right\|_{m(\phi, p)} \\
& +\left\|\sum_{j=1}^{n}\left(\sum_{i=\tau_{j-1}+1}^{\tau_{j}} z_{j}(i) e(i)\right)\right\|_{m(\phi, p)} \\
\leq & \sum_{j=1}^{n}\left\|\left(\sum_{i=\tau_{j-1}+1}^{\tau_{j}} z_{j}(i) e(i)\right)\right\|_{m(\phi, p)}^{n}+2 \sum_{j=1} \epsilon_{j} .
\end{aligned}
$$


By using the norm of the space $m(\phi, p)$, we have

$$
\begin{gathered}
\sum_{j=1}^{n}\left\|\left(\sum_{i=\tau_{j-1}+1}^{\tau_{j}} z_{j}(i) e(i)\right)\right\|_{m(\phi, p)} \\
=\sum_{j=1}^{n} \sup _{\tau \geq 1} \sup _{s_{j-1} \in \mathscr{C}_{\tau}}\left(\frac{1}{\phi_{\tau}} \sum_{n \in s_{j-1}}\left|z_{j}(n)\right|^{p}\right) \\
\leq \sum_{j=1}^{n} \sup _{\tau \geq 1} \sup _{\sigma \in \mathscr{C}_{\tau}}\left(\frac{1}{\phi_{\tau}} \sum_{n \in \sigma}\left|z_{j}(n)\right|^{p}\right) \leq n .
\end{gathered}
$$

Therefore,

$$
\left\|\sum_{j=1}^{n} z_{j}\right\|_{m(\phi, p)} \leq n^{1 / p}+1 \leq 2 n^{1 / p}
$$

This completes the proof of the theorem.

Theorem 5. For $1<p<\infty$, the space $m(\phi, p)$ has the weak fixed point property, if $K>2^{1-p}$, where $K=\sup _{\tau \geq 1} \phi_{\tau}<\infty$.

Proof. If $\psi_{\tau}=1$, for all $\tau \in \mathbb{N}$, it follows that

$$
\begin{gathered}
m(\phi, p) \subseteq \ell_{p} \quad \text { iff } \sup _{\tau \geq 1}\left(\phi_{\tau}\right)^{1 / p}<\infty \\
\|x\|_{m(\phi, p)}=\sup _{\tau \geq 1}\left(\frac{1}{\phi_{\tau}}\right)^{1 / p}\|x\|_{\ell_{p}} .
\end{gathered}
$$

Hence

$$
\begin{aligned}
R(m(\phi, p)) & =\sup _{\tau \geq 1}\left(\frac{1}{\phi_{\tau}}\right)^{1 / p} R\left(\ell_{p}\right) \\
& =\left(\frac{2}{K}\right)^{1 / p} \\
& <2, \quad \text { since } R\left(\ell_{p}\right)=2^{1 / p},
\end{aligned}
$$

where $R(X)$ stands for the Garcia-Falset coefficient of $X$. Therefore, $m(\phi, p)$ has in this case the weak fixed point property.

\section{Banach-Saks Type and Gurariǐ Modulus of Sequence Spaces $\ell_{p}(u, v)$}

Theorem 6. The space $\ell_{p}(u, v)$ has the Banach-Saks property of the type $p$.

Proof. Let $\left(\varepsilon_{n}\right)$ be a sequence of positive numbers for which $\sum_{n=1}^{\infty} \varepsilon_{n} \leq(1 / 2)$. Let $\left\{x_{n}\right\}$ be a weakly null sequence in $B\left(\ell_{p}(u, v)\right)$. Set $t_{0}=x_{0}=0$ and $t_{1}=x_{n_{1}}=x_{1}$. Then there exists $r_{1} \in \mathbb{N}$ such that

$$
\left\|\sum_{i=r_{1}+1}^{\infty} t_{1}(i) e(i)\right\|_{\ell_{p}(u, v)}<\varepsilon_{1} .
$$

Since the fact that $\left\{x_{n}\right\}$ is a weakly null sequence implies that $x_{n} \rightarrow 0$, coordinatewise, there is an $n_{2} \in \mathbb{N}$ such that

$$
\left\|\sum_{i=1}^{r_{1}} x_{n}(i) e(i)\right\|_{\ell_{p}(u, v)}<\varepsilon_{1}
$$

for all $n \geq n_{2}$. Set $t_{2}=x_{n_{2}}$. Then there exists an $r_{2}>r_{1}$ such that

$$
\left\|\sum_{i=r_{2}+1}^{\infty} t_{2}(i) e(i)\right\|_{\ell_{p}(u, v)}<\varepsilon_{2} .
$$

By using the fact that $x_{n} \rightarrow 0$ coordinatewise, there exists an $n_{3}>n_{2}$ such that

$$
\left\|\sum_{i=1}^{r_{2}} x_{n}(i) e(i)\right\|_{\ell_{p}(u, v)}<\varepsilon_{2}
$$

for all $n \geq n_{3}$. Continuing this process, we can find by induction two increasing subsequences $\left(r_{i}\right)$ and $\left(n_{i}\right)$ of natural numbers such that

$$
\left\|\sum_{i=1}^{r_{j}} x_{n}(i) e(i)\right\|_{\ell_{p}(u, v)}<\varepsilon_{j},
$$

for all $n \geq n_{j+1}$ and

$$
\left\|\sum_{i=r_{j}+1}^{\infty} t_{j}(i) e(i)\right\|_{\ell_{p}(u, v)}<\varepsilon_{j},
$$

where $t_{j}=x_{n_{j}}$. Hence,

$$
\begin{aligned}
\left\|\sum_{j=0}^{n} t_{j}\right\|_{\ell_{p}(u, v)}= & \| \sum_{j=0}^{n}\left(\sum_{i=0}^{r_{j-1}} t_{j}(i) e(i)+\sum_{i=r_{j-1}+1}^{r_{j}} t_{j}(i) e(i)\right. \\
& \left.+\sum_{i=r_{j}+1}^{\infty} t_{j}(i) e(i)\right) \|_{\ell_{p}(u, v)} \\
\leq & \left\|\sum_{j=0}^{n}\left(\sum_{i=r_{j-1}+1}^{r_{j}} t_{j}(i) e(i)\right)\right\|_{\ell_{p}(u, v)} \\
& +\left\|\sum_{j=0}^{n}\left(\sum_{i=0}^{r_{j-1}} t_{j}(i) e(i)\right)\right\|_{\ell_{p}(u, v)} \\
& +\left\|\sum_{j=0}^{n}\left(\sum_{i=r_{j}+1}^{\infty} t_{j}(i) e(i)\right)\right\|_{\ell_{p}(u, v)} \\
\leq & \left\|\sum_{j=0}^{n}\left(\sum_{i=r_{j-1}+1}^{r_{j}} t_{j}(i) e(i)\right)\right\|_{\ell_{p}(u, v)}+2 \sum_{j=0}^{n} \varepsilon_{j} .
\end{aligned}
$$


On the other hand, since $\left\|x_{n}\right\|=\left(\sum_{i=0}^{\infty}\left|\sum_{k=0}^{i} u_{i} v_{k} x_{n_{j}}(k)\right|^{p}\right)^{1 / p}$, it can be easily seen that $\left\|x_{n}\right\|<1$. Therefore, $\left\|x_{n}\right\|^{p}<1$ and

$$
\begin{aligned}
& \left\|\sum_{j=0}^{n}\left(\sum_{i=r_{j-1}+1}^{r_{j}} t_{j}(i) e(i)\right)\right\|_{\ell_{p}(u, v)}^{p} \\
& =\sum_{j=0}^{n} \sum_{i=r_{j-1}+1}^{r_{j}}\left|\sum_{k=0}^{i} u_{i} v_{k} t_{j}(k)\right|^{p} \\
& \leq \sum_{j=0}^{n} \sum_{i=0}^{\infty}\left|\sum_{k=0}^{i} u_{i} v_{k} t_{j}(k)\right|^{p} \leq(n+1) .
\end{aligned}
$$

Hence we obtain

$$
\begin{gathered}
\left\|\sum_{j=0}^{n}\left(\sum_{i=r_{j-1}+1}^{r_{j}} t_{j}(i) e(i)\right)\right\| \\
\leq\left(\sum_{j=0}^{n} 1\right)^{1 / p}=(n+1)^{1 / p} .
\end{gathered}
$$

By using the inequality $1 \leq(n+1)^{1 / p}$ for all $n \in \mathbb{N}$ and $1 \leq$ $p<\infty$, we have

$$
\left\|\sum_{j=0}^{n} t_{j}\right\|_{l_{p}(u, v)} \leq(n+1)^{1 / p}+1 \leq 2(n+1)^{1 / p} .
$$

Therefore, the space $\ell_{p}(u, v)$ has the Banach-Saks type $p$, which completes the proof of the theorem.

$$
\text { Let us define the matrix } G=G(u, v)=\left\{g_{n k}\right\} \text { by }
$$

$$
g_{n k}= \begin{cases}u_{n} v_{k} ; & 0 \leq k \leq n \\ 0 ; & k>n\end{cases}
$$

for all $k, n \in \mathbb{N}$, where $u_{n}$ depends only on $n$ and $v_{k}$ depends only on $k$. The matrix $G$ is called generalized weighted mean or factorable matrix. By $H=H(v, u)=\left(h_{n k}\right)$, we denote the inverse of the matrix $G(u, v)$ as follows:

$$
h_{n k}= \begin{cases}\frac{(-1)^{n-k}}{v_{n} u_{k}} ; & n-1 \leq k \leq n, \\ 0 ; & 0 \leq n<k \text { or } n>k+1 .\end{cases}
$$

Theorem 7. For $x \in \ell_{p}(u, v)$, by (39), one has the fact that the Gurari modulus of convexity for the normed space $\ell_{p}(u, v)$ satisfies the inequality

$$
\beta_{\ell_{p}(u, v)}(\varepsilon) \leq 1-\left(1-\left(\frac{\varepsilon}{2}\right)^{p}\right)^{1 / p}
$$

for any $0 \leq \varepsilon \leq 2$.
Proof. Let $x \in \ell_{p}(u, v)$. By using (39), we have

$$
\begin{aligned}
\|x\|_{\ell_{p}(u, v)} & =\|G(u, v) x\|_{\ell_{p}} \\
& =\left(\sum_{n}\left|(G(u, v) x)_{n}\right|^{p}\right)^{1 / p} .
\end{aligned}
$$

Let $0 \leq \varepsilon \leq 2$. Then using (40), let us consider the following sequences:

$$
\begin{aligned}
& x=\left(x_{n}\right)=\left(H\left(\left(1-\left(\frac{\varepsilon}{2}\right)^{p}\right)^{1 / p}\right), H\left(\frac{\varepsilon}{2}\right), 0,0, \ldots\right), \\
& t=\left(t_{n}\right)=\left(H\left(\left(1-\left(\frac{\varepsilon}{2}\right)^{p}\right)^{1 / p}\right), H\left(-\frac{\varepsilon}{2}\right), 0,0, \ldots\right) .
\end{aligned}
$$

Since $y_{n}=(G x)_{n}$ and $z_{n}=(G t)_{n}$, we have

$$
\begin{aligned}
& y=\left(y_{n}\right)=\left(\left(1-\left(\frac{\varepsilon}{2}\right)^{p}\right)^{1 / p},\left(\frac{\varepsilon}{2}\right), 0,0, \ldots\right) \\
& z=\left(z_{n}\right)=\left(\left(1-\left(\frac{\varepsilon}{2}\right)^{p}\right)^{1 / p},\left(-\frac{\varepsilon}{2}\right), 0,0, \ldots\right)
\end{aligned}
$$

By using the sequences given above, we obtain the following equalities:

$$
\begin{aligned}
&\|x\|_{\ell_{p}(u, v)}^{p}=\|G(u, v) x\|_{\ell_{p}}^{p} \\
&=\left|\left(1-\left(\frac{\varepsilon}{2}\right)^{p}\right)^{1 / p}\right|^{p}+\left|\frac{\varepsilon}{2}\right|^{p} \\
&= 1-\left(\frac{\varepsilon}{2}\right)^{p}+\left(\frac{\varepsilon}{2}\right)^{p}=1 ; \\
&\|t\|_{\ell_{p}(u, v)=}^{p}=\|G(u, v) t\|_{\ell_{p}}^{p} \\
&=\left|\left(1-\left(\frac{\varepsilon}{2}\right)^{p}\right)^{1 / p}\right|^{p}+\left|-\frac{\varepsilon}{2}\right|^{p} \\
&= 1-\left(\frac{\varepsilon}{2}\right)^{p}+\left(\frac{\varepsilon}{2}\right)^{p}=1 ; \\
&\|x-t\|_{\ell_{p}(u, v)=}\|G(u, v) x-G(u, v) t\|_{\ell_{p}} \\
&=\left(\left|\left(1-\left(\frac{\varepsilon}{2}\right)^{p}\right)^{1 / p}-\left(1-\left(\frac{\varepsilon}{2}\right)^{p}\right)^{1 / p}\right|^{p}\right. \\
&\left.+\left|\frac{\varepsilon}{2}-\left(-\frac{\varepsilon}{2}\right)\right|^{p}\right)^{1 / p}=\varepsilon .
\end{aligned}
$$


To complete the upper estimate of the Gurarii modulus of convexity, it remains to calculate the infimum of $\|\alpha x+(1-\alpha) t\|_{\ell_{p}(u, v)}$ for $0 \leq \alpha \leq 1$. We have

$$
\begin{aligned}
& \inf _{0 \leq \alpha \leq 1}\|\alpha x+(1-\alpha) t\|_{\ell_{p}(u, v)} \\
&= \inf _{0 \leq \alpha \leq 1}\|\alpha G(u, v) x+(1-\alpha) G(u, v) t\|_{\ell_{p}} \\
&= \inf _{0 \leq \alpha \leq 1}\left[\mid \alpha\left(1-\left(\frac{\varepsilon}{2}\right)^{p}\right)^{1 / p}\right. \\
&+\left.(1-\alpha)\left(1-\left(\frac{\varepsilon}{2}\right)^{p}\right)^{1 / p}\right|^{p} \\
&\left.+\left|\alpha\left(\frac{\varepsilon}{2}\right)+(1-\alpha)\left(-\frac{\varepsilon}{2}\right)\right|^{p}\right]^{1 / p} \\
&= \inf _{0 \leq \alpha \leq 1}\left[1-\left(\frac{\varepsilon}{2}\right)^{p}+|2 \alpha-1|^{p}\left(\frac{\varepsilon}{2}\right)^{p}\right]^{1 / p} \\
&=\left(1-\left(\frac{\varepsilon}{2}\right)^{p}\right)^{1 / p} .
\end{aligned}
$$

Consequently, we get for $p \geq 1$ the inequality

$$
\beta_{l_{p}(u, v)}(\varepsilon) \leq 1-\left(1-\left(\frac{\varepsilon}{2}\right)^{p}\right)^{1 / p},
$$

which is the desired result.

\section{Conflict of Interests}

The authors declare that there is no conflict of interests regarding the publication of this paper.

\section{References}

[1] G. Alherk and H. Hudzik, "Uniformly non- $l_{n}^{(1)}$ Musielak-Orlicz spaces of Bochner type," Forum Mathematicum, vol. 1, no. 4, pp. 403-410, 1989.

[2] G. Alherk and H. Hudzik, "Copies of $l^{1}$ and $c_{0}$ in MusielakOrlicz sequence spaces," Commentationes Mathematicae Universitatis, vol. 35, no. 1, pp. 9-19, 1994.

[3] L. X. Bo, Y. A. Cui, P. Foralewski, and H. Hudzik, "Local uniform rotundity and weak local uniform rotundity of Musielak-Orlicz sequence spaces endowed with the Orlicz norm," Nonlinear Analysis, Theory, Methods and Applications, vol. 69, no. 5-6, pp. 1559-1569, 2008.

[4] Y. A. Cui, H. Hudzik, and R. Płuciennik, "Banach-Saks property in some Banach sequence spaces," Annales Polonici Mathematici, vol. 65, no. 2, pp. 193-202, 1997.

[5] Y. A. Cui, H. Hudzik, N. Petrot, S. Suantai, and A. Szymaszkiewicz, "Basic topological and geometric properties of Cesàro-Orlicz spaces," Proceedings of the Indian Academy of Sciences, vol. 115, no. 4, pp. 461-476, 2005.
[6] Y. A. Cui, H. Hudzik, M. Wisła, and M. Zou, "Extreme points and strong U-points in Musielak-Orlicz sequence spaces equipped with the Orlicz norm," Zeitschrift fur Analysis und ihre Anwendung, vol. 26, no. 1, pp. 87-101, 2007.

[7] M. Denker and H. Hudzik, "Uniformly non- $l_{n}^{(1)}$ MusielakOrlicz sequence spaces," Proceedings of the Indian Academy of Sciences, vol. 101, no. 2, pp. 71-86, 1991.

[8] F. Fuentes and F. L. Hernandez, "On weighted Orlicz sequence spaces and their subspaces," Rocky Mountain Journal of Mathematics, vol. 18, pp. 585-599, 1988.

[9] H. Hudzik, "Uniformly non- $l_{n}^{(1)}$ Orlicz spaces with Luxemburg norm," Studia Mathematica, vol. 81, no. 3, pp. 271-284, 1985.

[10] H. Hudzik and D. Pallaschke, "On some convexity properties of Orlicz sequence spaces equipped with the Luxemburg norm," Mathematische Nachrichten, vol. 186, pp. 167-185, 1997.

[11] H. Hudzik and Y. N. Ye, "Support functionals and smoothness in Musielak-Orlicz sequence spaces endowed with the Luxemburg norm," Commentationes Mathematicae Universitatis Carolinae, vol. 031, no. 4, pp. 661-684, 1990.

[12] H. Hudzik and L. Szymaszkiewicz, "Basic topological and geometric properties of orlicz spaces over an arbitrary set of atoms," Zeitschrift fur Analysis und ihre Anwendung, vol. 27, no. 4, pp. 425-449, 2008.

[13] J. E. Jamison, A. Kamińska, and G. Lewicki, "One-complemented subspaces of Musielak-Orlicz sequence spaces," Journal of Approximation Theory, vol. 130, no. 1, pp. 1-37, 2004.

[14] A. Kamińska, "Strict convexity of sequence Orlicz-Musielak spaces with Orlicz norm," Journal of Functional Analysis, vol. 50, no. 3, pp. 285-305, 1983.

[15] A. Kamińska, "Uniform rotundity of Musielak-Orlicz sequence spaces," Journal of Approximation Theory, vol. 47, no. 4, pp. 302322, 1986.

[16] A. Kamińska, "Flat Orlicz-Musielak sequence spaces," Bulletin de L'Academie Polonaise des Sciences, vol. 29, pp. 137-144, 1981.

[17] A. Kamińska and L. Han Ju, "Banach-Saks properties of Musielak-Orlicz and Nakano sequence spaces," Proceedings of the American Mathematical Society, vol. 142, pp. 547-558, 2014.

[18] A. Kamińska, "Uniform rotundity in every direction of sequence Orlicz spaces," Bulletin de L'Academie Polonaise des Sciences, vol. 32, pp. 589-594, 1984.

[19] A. Kamińska, "Rotundity of sequence Musielak-Orlicz spaces," Bulletin de L'Academie Polonaise des Sciences, vol. 29, pp. 137144,1981

[20] V. Karakaya, "Some geometric properties of sequence spaces involving lacunary sequence," Journal of Inequalities and Applications, vol. 2007, Article ID 81028, 2007.

[21] E. Katirtzoglou, "Type and Cotype of Musielak-Orlicz Sequence Spaces," Journal of Mathematical Analysis and Applications, vol. 226, no. 2, pp. 431-455, 1998.

[22] H. Knaust, "Orlicz sequence spaces of Banach-Saks type," Archiv der Mathematik, vol. 59, no. 6, pp. 562-565, 1992.

[23] P. Kolwicz, "Strictly and uniformly monotone sequential Musielak-Orlicz spaces, Fourth International Conference on Function Spaces (Zielona Gora, 1995)," Collectanea Mathematica, vol. 50, no. 1, pp. 1-17, 1999.

[24] W. Kurc, "Strictly and uniformly monotone sequential Musielak-Orlicz spaces," Collectanea Mathematica, vol. 50, no. 1, pp. 1-17, 1999.

[25] V. Peirats and C. Ruiz, "On $l_{p}$-copies in Musielak-Orlicz sequence spaces," Archiv der Mathematik, vol. 58, no. 2, pp. 164$173,1992$. 
[26] Y. Cui and H. Hudzik, "Packing constant for Cesàro sequence spaces, proceedings of the third world congress of nonlinear analysis, part 4 (Catania, 2000)," Nonlinear Analysis, Theory, Methods and Applications, vol. 47, no. 4, pp. 2695-2702, 2001.

[27] Y. A. Cui and H. Hudzik, "Some geometric properties related to fixed point theory in Cesàro spaces," Collectanea Mathematica, vol. 50, no. 3, pp. 277-288, 1999.

[28] Y. A. Cui and H. Hudzik, "On the Banach-Saks and weak Banach-Saks properties of some Banach sequence spaces," Acta Scientiarum Mathematicarum, vol. 65, no. 1-2, pp. 179-187, 1999.

[29] Y. A. Cui and H. Hudzik, "Packing constant for Cesàro sequence spaces," Nonlinear Analysis, Theory, Methods and Applications, vol. 47, no. 4, pp. 2695-2702, 2001.

[30] Y. A. Cui and C. Meng, "Banach-Saks property and property $(\beta)$ in Cesàro sequence spaces," Southeast Asian Bulletin of Mathematics, vol. 24, pp. 201-210, 2000.

[31] S. Suantai, "In the H-property of some Banach sequence spaces," Archivum Mathematicum, vol. 39, pp. 309-316, 2003.

[32] P. N. Ng and P. Y. Lee, "Cesàro sequences spaces of non-absolute type," Commentationes Mathematicae, vol. 20, no. 2, pp. 429433, 1978.

[33] P. Foralewski, H. Hudzik, and A. Szymaszkiewicz, "Local rotundity structure of Cesàro-Orlicz sequence spaces," Journal of Mathematical Analysis and Applications, vol. 345, no. 1, pp. 410-419, 2008.

[34] P. Foralewski, H. Hudzik, and A. Szymaszkiewicz, "Some remarks on Cesàro-Orlicz sequence spaces," Mathematical Inequalities and Applications, vol. 13, no. 2, pp. 363-386, 2010.

[35] A. Kamińska and D. Kubiak, "On isometric copies of $l_{\infty}$ and James constants in Cesàro-Orlicz sequence spaces," Journal of Mathematical Analysis and Applications, vol. 372, no. 2, pp. 574584, 2010.

[36] D. Kubiak, "A note on Cesàro-Orlicz sequence spaces," Journal of Mathematical Analysis and Applications, vol. 349, no. 1, pp. 291-296, 2009.

[37] P. Foralewski, H. Hudzik, and L. Szymaszkiewicz, "Local rotundity structure of generalized Orlicz-Lorentz sequence spaces," Nonlinear Analysis, Theory, Methods and Applications, vol. 68, no. 9, pp. 2709-2718, 2008.

[38] P. Foralewski, H. Hudzik, and P. Kolwicz, "Non-squareness properties of Orlicz-Lorentz sequence spaces," Journal of Functional Analysis, vol. 264, no. 2, pp. 605-629, 2013.

[39] M. Mursaleen, F. Başar, and B. Altay, "On the Euler sequence spaces which include the spaces $\ell \mathrm{p}$ and $\ell_{\infty}$ II," Nonlinear Analysis, Theory, Methods and Applications, vol. 65, no. 3, pp. 707-717, 2006.

[40] H. Hudzik and A. Narloch, "Relationships between monotonicity and complex rotundity properties with some consequences," Mathematica Scandinavica, vol. 96, no. 2, pp. 289-306, 2005.

[41] P. Kolwicz and R. Płuciennik, "Points of upper local uniform monotonicity in Calderón-Lozanovskii spaces," Journal of Convex Analysis, vol. 17, no. 1, pp. 111-130, 2010.

[42] P. Kolwicz and K. Leśnik, "Property $\beta$ of Rolewicz and orthogonal convexities of Calderón-Lozanovskiǐ spaces," Nonlinear Analysis, Theory, Methods and Applications, vol. 74, no. 13, pp. 4352-4368, 2011.

[43] P. Kolwicz, "Kadec-Klee properties of Calderón-Lozanovskiǐ sequence spaces," Collectanea Mathematica, vol. 63, no. 1, pp. 45-58, 2012.

[44] B. Altay and F. Başar, "On the paranormed Riesz sequence spaces of non-absolute type," Southeast Asian Bulletin of Mathematics, vol. 26, pp. 701-715, 2002.
[45] B. Altay and F. Başar, "Generalization of the sequence space $l(p)$ derived by weighted mean," Journal of Mathematical Analysis and Applications, vol. 330, no. 1, pp. 174-185, 2007.

[46] P. Kolwicz, "Property $(\beta)$ and orthogonal convexities income class of Köthe sequence spaces," Publicationes Mathematicae, vol. 63, no. 4, pp. 587-609, 2003.

[47] H. Hudzik and P. Kolwicz, "On property $(\beta)$ of Rolewicz in Köthe-Bochner sequence spaces," Studia Mathematica, vol. 162, no. 3, pp. 195-212, 2004.

[48] I. J. Maddox, "Paranormed sequence spaces generated by infinite matrices," Proceedings-Cambridge Philosophical Society, vol. 64, pp. 335-340, 1968.

[49] E. Malkowsky and M. Mursaleen, "Matrix transformations between $F K$-spaces and the sequence spaces $m(\phi)$ and $n(\phi)$," Journal of Mathematical Analysis and Applications, vol. 196, no. 2, pp. 659-665, 1995.

[50] E. Malkowsky and M. Mursaleen, "Compact matrix operators between the spaces $m(\phi), n(\phi)$ and $l_{p}$," Bulletin of the Korean Mathematical Society, vol. 48, no. 5, pp. 1093-1103, 2011.

[51] E. Malkowsky and E. Savas, "Matrix transformations between sequence spaces of generalized weighted means," Applied Mathematics and Computation, vol. 147, no. 2, pp. 333-345, 2004.

[52] M. Mursaleen, "Some geometric proprties of a sequence space related to $l^{p}$, , Bulletin of the Australian Mathematical Society, vol. 67, no. 2, pp. 343-347, 2003.

[53] M. Mursaleen, Elements of Metric Spaces, Anamaya, New Delhi, India, 2005.

[54] M. Mursaleen, R. Çolak, and M. Et, "Some geometric inequalities in a new Banach sequence space," Journal of Inequalities and Applications, vol. 2007, Article ID 86757, 6 pages, 2007.

[55] W. L. C. Sargent, "Some sequence spaces related to the $l^{p}$ spaces," Journal of the London Mathematical Society, vol. 35, pp. 161-171, 1960.

[56] S. Simons, "The sequence spaces $l\left(p_{v}\right)$ and $m\left(p_{v}\right)$," Proceedings of the London Mathematical Society, vol. 15, no. 3, pp. 422-436, 1965.

[57] N. Şimşek and V. Karakaya, "On some geometrial properties of generalized modular spaces of Cesàro type defined by weighted means," Journal of Inequalities and Applications, vol. 2009, Article ID 932734, 13 pages, 2009.

[58] E. Savaş, V. Karakaya, and N. Şimşek, "Some $l(p)$-Type new sequence spaces and their geometric properties," Abstract and Applied Analysis, vol. 2009, Article ID 696971, 12 pages, 2009.

[59] N. Şimşek, E. Savas, and V. Karakaya, “On geometrical properties of some banach spaces," Applied Mathematics \& Information Sciences, vol. 7, no. 1, pp. 295-300, 2013.

[60] C. S. Wang, “On Nörlund sequence space," Tamkang Journal of Mathematics, vol. 9, pp. 269-274, 1978.

[61] B. C. Tripathy and M. Sen, "On a new class of sequences related to the space $l^{p}$," Tamkang Journal of Mathematics, vol. 33, no. 2, pp. 167-171, 2002.

[62] J. Y. T. Woo, “On Modular Sequence spaces," Studia Mathematica, vol. 48, pp. 271-289, 1973.

[63] J. Diestel, "Sequence and series in Banach spaces," in Graduate Texts in Math, vol. 92, Springer, New York, NY, USA, 1984.

[64] J. Garcia-Falset, "Stability and fixed points for nonexpansive mappings," Houston Journal of Mathematics, vol. 20, pp. 495505, 1994.

[65] J. G. Falset, "The fixed point property in Banach spaces with the NUS-property," Journal of Mathematical Analysis and Applications, vol. 215, no. 2, pp. 532-542, 1997. 
[66] J. A. Clarkson, "Uniformly convex spaces," Transactions of the American Mathematical Society, vol. 40, pp. 396-414, 1936.

[67] M. M. Day, "Uniform convexity in factor and conjugate spaces," Annals of Mathematics, vol. 45, no. 2, pp. 375-385, 1944.

[68] V. I. Gurariǐ, "On moduli of convexity and attering of Banach spaces," Soviet Mathematics Doklady, vol. 161, no. 5, pp. 10031006, 1965.

[69] V. I. Gurariǐ, "On differential properties of the convexity moduli of Banach spaces," Matematicheskie Issledovaniya, vol. 2, pp. 141148, 1969.

[70] N. I. Gurariǐ and Y. U. Sozonov, "Normed spaces that do not have distortion of the unit sphere," Matematicheskie Zametki, vol. 7, pp. 307-310, 1970 (Russian).

[71] C. Zanco and A. Zucchi, "Moduli of rotundity and smoothness for convex bodies," Bolletino della Unione Matematica Italiana $B$, vol. 7, no. 7, pp. 833-855, 1993. 


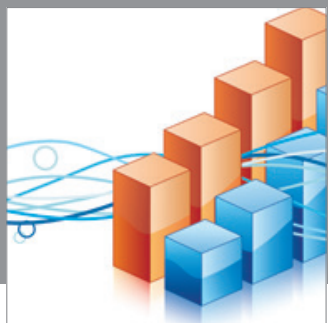

Advances in

Operations Research

mansans

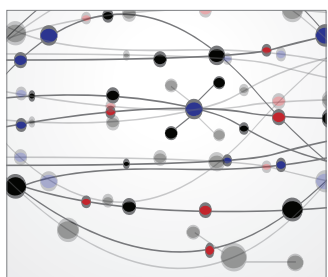

The Scientific World Journal
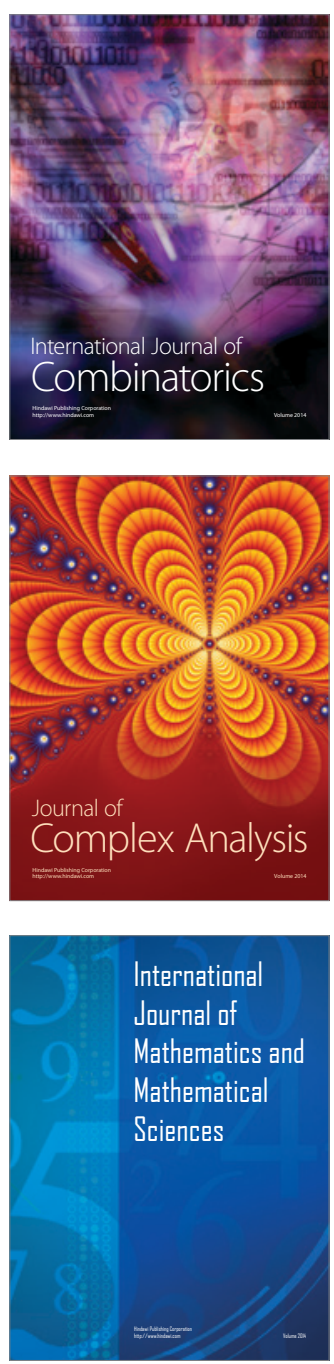
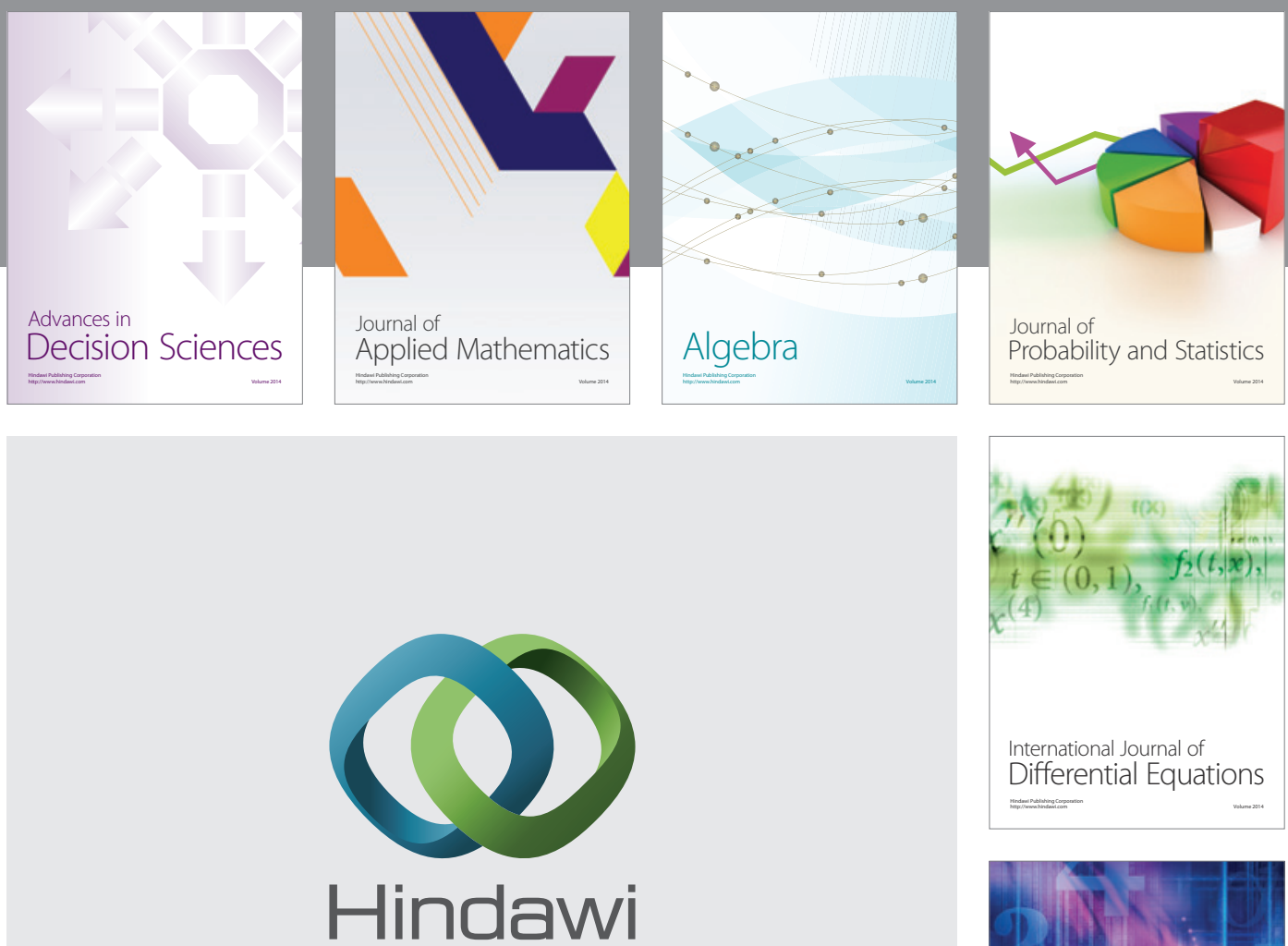

Submit your manuscripts at http://www.hindawi.com
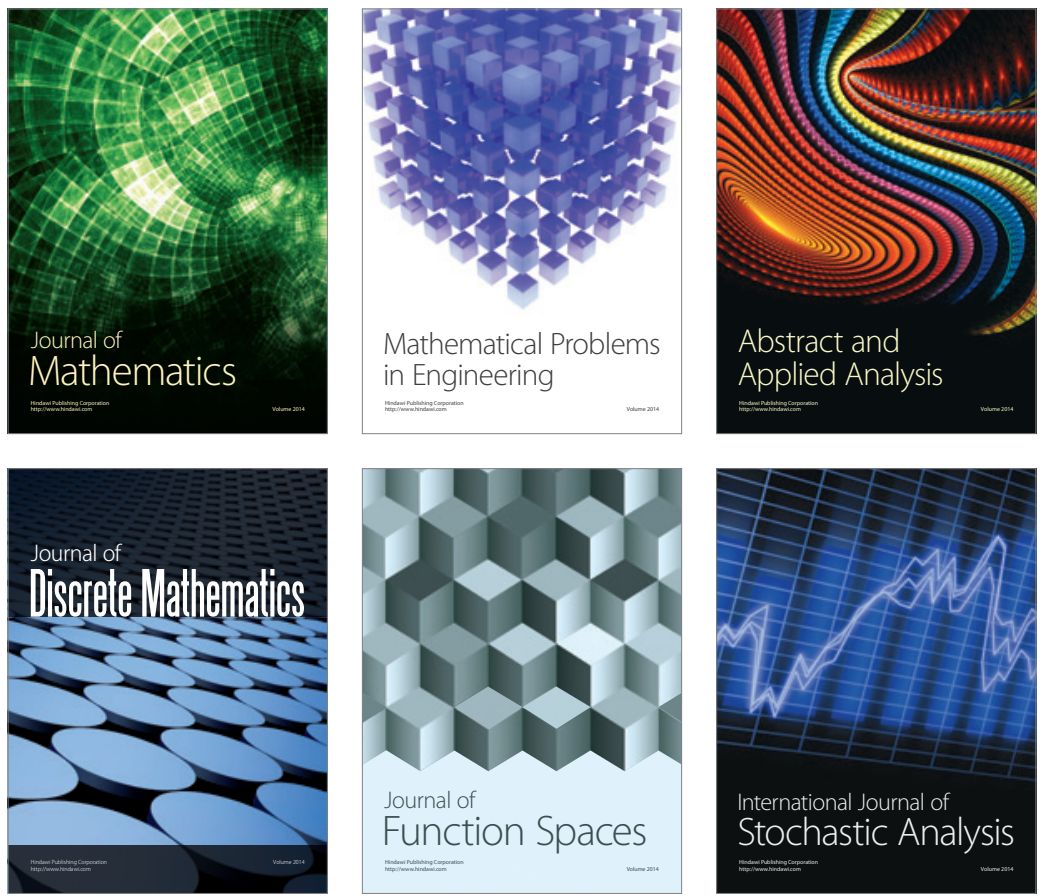

Journal of

Function Spaces

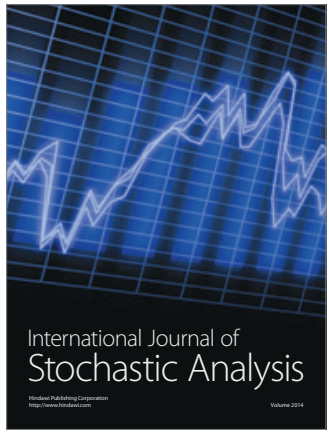

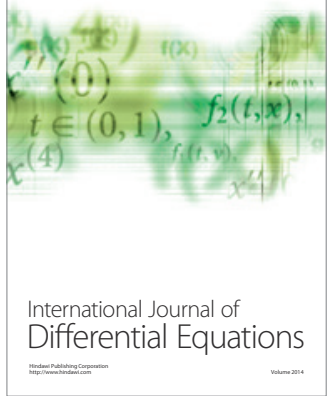
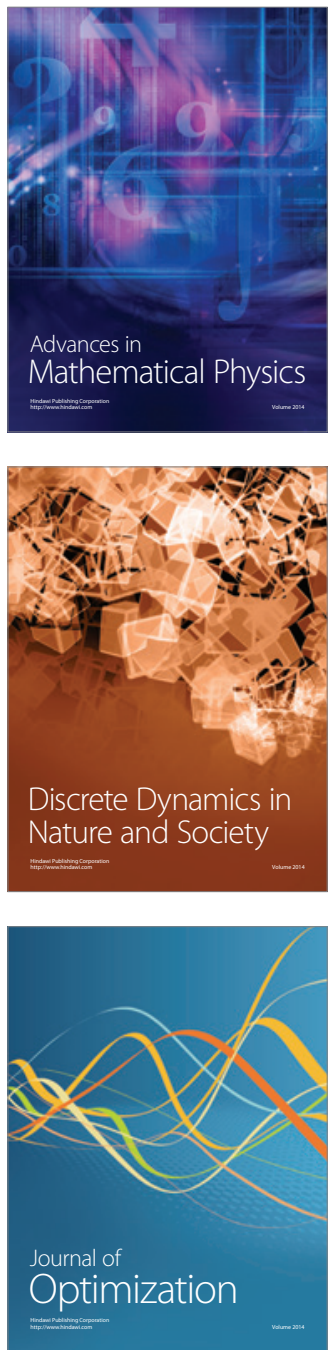